\title{
Unified approach for cluster variation method calculations of phase diagrams in fcc substitutional alloys with interstitial species
}

\author{
Diana E. Nanu, Youjin Deng,* and Amarante J. Böttger ${ }^{\dagger}$ \\ Department of Materials Science and Engineering, Delft University of Technology, Mekelweg 2, 2628 CD Delft, The Netherlands
}

(Received 29 March 2006; published 31 July 2006)

\begin{abstract}
Some alloys show interstitial-induced phase transitions and order-disorder transitions due to the mutual interactions between the interstitial $(I)$ species and the substitutional $(S)$ host lattice. An innovative approach, based on the cluster variation method (CVM), that takes this coupling into account is proposed here for the calculation of thermodynamic data and phase boundaries. In the case of fcc substitutional alloys with interstitial species a simple cube is chosen as the basic cluster. The cube is defined such that it explicitly accounts for the mutual interaction between the $S$ and the $I$ sublattices comprising the system. Expressions for the configurational entropy in the cube approximation and the internal energy are derived. Phase diagrams for several hypothetical binary host alloys with interstitials are calculated. The results obtained using the proposed simplecube approximation demonstrate the effect of mutual interactions on the phase boundaries.
\end{abstract}

DOI: 10.1103/PhysRevB.74.014113

PACS number(s): 64.60.Cn, 82.60.Lf, 64.70.Kb

\section{INTRODUCTION}

The thermodynamics and phase stability of interstitial systems is important since such alloys are encountered in numerous industrial applications: nitrogen and carbon in steels, boron or carbon in aluminum alloys, and hydrogen in palladium-based membranes or in materials for hydrogen storage. These systems consist of a host metal lattice of which interstitial sites are partly occupied by other atomic species. In most of the aforementioned examples, the metal lattice is a substitutional metal alloy. On both sublattices, the substitutional host ( $S$ sublattice) and the interstitial sublattice (I sublattice), order-disorder transitions could occur. Moreover the presence of interstitial atoms could induce ordering in the substitutional metal lattice, or vice versa, the presence of substitutional atoms in the metal lattice could induce ordering of the interstitial atoms. Experiments indicate that in a number of Pd-alloys (e.g., those alloyed with metals from group III $b$ - $\mathrm{V} b$, and with rare earth metals) hydrogen-induced ordering occurs. ${ }^{1-3}$ Also other systems show ordering transitions induced by the presence of interstitial atoms, e.g., Fe$\mathrm{Cr}-\mathrm{C}, \mathrm{Fe}-\mathrm{Cr}-\mathrm{N}, \mathrm{Al}-\mathrm{C}-\mathrm{Mn}$, and Al-B-Mn. ${ }^{4} \mathrm{~A}$ sound thermodynamic model for such systems should be capable of predicting the presence of short- and (or) long-range order, as well as the occurrence of order-disorder transformations. In this paper a unified approach, i.e., one that incorporates the coupling of the interstitial and substitutional lattices, is put forward.

The cluster variation method (CVM) (Refs. 5-9) is used to describe the substitutional-interstitial systems as it has been recognized to provide a reliable way to estimate the configurational entropy and to predict thermodynamic properties and phase transformations in systems that show orderdisorder transformations. ${ }^{10-16}$ The main approximation in the CVM is the choice of a basic cluster up to which the atomic correlations are accounted for in the free energy functional. Recently, the combination of CVM with ab initio calculations has opened the path towards a priori predictions of phase diagrams in solid systems. Most of these studies, however, deal with binary or ternary substitutional alloys, and only few applications to interstitial solid solutions have been reported. ${ }^{17-20} \mathrm{Up}$ to now, the distribution of interstitial atoms on the $I$ sublattice is described as that of a binary (or ternary) interstitial atom(s)-vacancy substitutional system in the mean-field created by the metal sublattice. ${ }^{17}$ Such an approach provides good results when the metal sublattice is a pure substance, ${ }^{17-20}$ but cannot be used to describe the influence of the atomic arrangement of the substitutional metal alloy on that of the interstitial atoms on the $I$ sublattice. The coupling between the two sublattices is not accounted for.

In this paper, a CVM approach that treats explicitly the coupling between the $S$ and the $I$ sublattices is proposed for calculations of thermodynamic data and phase boundaries of fcc substitutional alloys with interstitial species. A simple cube is chosen as the basic cluster. The cube is defined such that it includes sites belonging to both the substitutional and the interstitial sublattices comprising the system. Expressions of the configurational entropy in the cube approximation and the internal energy are derived in Sec. II. The proposed approach is then applied to calculate phase boundaries for several hypothetical binary host alloys with interstitials. The results, discussed in Sec. III, illustrate that the proposed approach can describe the mutual interaction of the $S$ and $I$ sublattices and its effect on the phase boundaries.

\section{CVM CUBE APPROXIMATION}

In this paper, binary fcc substitutional alloys that contain interstitial atoms are considered. It is assumed that the interstitial atoms only occupy the octahedral sites formed by the close-packed metal lattice. Hence the interstitial lattice has also a fcc structure. The sites of the substitutional host sublattice and the sites belonging to the interstitial sublattice are denoted by $S$ and $I$, respectively. For convenience it is considered that the $S$ and $I$ lattice sites can both be occupied by two types of species (atoms or vacancies), as follows: species $A_{1}$ and $A_{2}$ on the $S$ sublattice, and $B_{1}$ and $B_{2}$ on the $I$ sublattice. The $A$ and $B$ types of species are restricted to occupy their own sublattices, i.e., $A$ species only reside on the $S$ sublattice, and $B$ species only reside on the $I$ sublattice. 


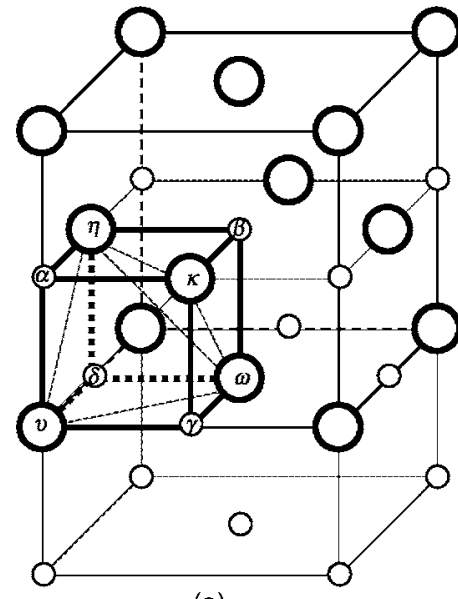

(a)

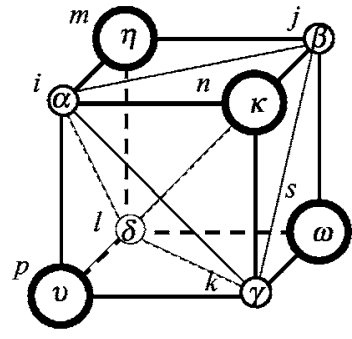

(b)
FIG. 1. Graphical representation of a fcc substitutional system with interstitial species. The sites on the $S$ and $I$ sublattices are represented by large and small open circles, respectively. The four neighboring species occupying the $S$ or $I$ sublattices of the fcc unit cell form a tetrahedron; two tetrahedrons from the $S$ and $I$ sublattices construct a simple cube, whose vertices are denoted by $\alpha, \beta$, $\gamma, \delta \eta, \kappa, v$, and $\omega$.

Such a system of two interpenetrating fcc lattices can be described by a simple cube as the basic cluster. The cube cluster is defined such that it includes sites from both the $S$ and $I$ sublattices (Fig. 1). In fact, as shown in Fig. 1, the simple cube consists of two tetrahedrons, one on the $S$ sublattice and the other on the $I$ sublattice.

The lattice sites on the cube are denoted by $\alpha, \beta, \gamma$, and $\delta$ for the $I$ sublattice and $\eta, \kappa, v$, and $\omega$ for the $S$ sublattice (Fig. 1). The occupation of the sites on the $I$ sublattice is described respectively by $i, j, k$, and $l$, which can take the values of 1 or 2 whether the sites are occupied by $B_{1}$ or $B_{2}$, while the occupation of the sites on the $S$ sublattice is described respectively by $m, n, p$, and $s$, which can take the values of 1 or 2 whether $A_{1}$ or $A_{2}$ occupies the sites. The cluster distribution variables of the simple cube are represented by $C_{i j k l m n p s}^{\alpha \beta \gamma \delta \eta \kappa v \omega}$. The super- and subscripts of $C_{i j k l m n p s}^{\alpha \beta \gamma \delta \eta \omega}$ specify the index of an element of an array of $2^{8}(256)$ elements, which represents all possible arrangements of four species on two separate sublattices. The simple-cube distribution variables obey the normalized condition

$$
\sum_{i j k l m n p s} C_{i j k l m n p s}^{\alpha \beta \gamma \delta \eta \kappa v \omega}=1
$$

where the summation is over all possible arrangements of species restricted to their sublattices.

The distribution variables of the subclusters included in the simple cube (i.e., point, pairs, etc.) are not independent of each other and can be obtained by summing the simple-cube cluster distribution variables $C_{i j k l m n p s}^{\alpha \beta \gamma \delta \eta \kappa \omega}$. For example, the distribution variables $W_{i j m n}^{\alpha \beta \eta \kappa}$ of the square cluster, $Y_{i m}^{\alpha \eta}$ of the nearest-neighbor pair cluster, and $X_{i}^{\alpha}$ or $X_{m}^{\eta}$ of the point cluster, can be calculated by Eq. (2). The summations in Eq. (2) involve all possible arrangements of the species over the specified lattice sites. For example, the square cluster $W_{i j m n}^{\alpha \beta \eta \kappa}$ consists of two lattice sites on the $I$ sublattice $(\alpha$ and $\beta$ ) and two on the $S$ sublattice ( $\eta$ and $\kappa$ ). Each of the $\alpha$ and $\beta$ sites can be occupied either by $B_{1}$ or $B_{2}$ (indexed by $i$ and $j$ ), and each of the $\eta$ and $\kappa$ sites can be occupied either by $A_{1}$ or $A_{2}$ (indexed by $m$ and $n$ ).

$$
\begin{gathered}
\text { Square: } W_{i j m n}^{\alpha \beta \eta \kappa}=\sum_{k l p s} C_{i j k l m n p s}^{\alpha \beta \gamma \delta \eta \kappa v \omega}, \ldots, \\
\text { Pair: } Y_{i m}^{\alpha \eta}=\sum_{j \mathrm{klnps}} C_{i j k l m n p s}^{\alpha \beta \gamma \delta \eta \kappa v \omega}=\sum_{\mathrm{jn}} W_{i j m n}^{\alpha \beta \eta \kappa}, \ldots, \\
\text { Point: } X_{i}^{\alpha}=\sum_{\mathrm{jklmnps}} C_{i j k l m n p s}^{\alpha \beta \gamma \delta \eta \kappa v \omega}=\sum_{\mathrm{m}} Y_{i m}^{\alpha \eta}, \ldots .
\end{gathered}
$$

Having defined the cluster distribution variables and their relations, one can proceed to define the thermodynamic functions describing the system in terms of the cluster distribution variables. The grand potential function, $\Omega$, is used to calculate the equilibrium states in this paper. For each phase, the grand potential per lattice site is defined ${ }^{21}$ as

$$
\begin{aligned}
& \Omega\left(V, T, \mu_{B_{1}}^{*}, \mu_{B_{2}}^{*}, \mu_{A_{1}}^{*}, \mu_{A_{2}}^{*}\right) \\
& =E-T S+p V-\frac{1}{2} \sum_{i=1}^{2} x_{i} \mu_{B_{i}}^{*}-\frac{1}{2} \sum_{m=1}^{2} y_{m} \mu_{A_{m}}^{*},
\end{aligned}
$$

where $E$ and $S$ describe the energy and the entropy per lattice site, respectively, $T$ is the temperature, $p$ is the external pressure, and $V$ represents the volume per lattice site. The terms $x_{i}$ and $y_{m}$ denote the mole fraction of component $B_{i}(i$ $=1,2)$ on the $I$ sublattice and of component $A_{m}(m=1,2)$ on the $S$ sublattice, respectively. The mole fractions are related to the point-cluster distribution variables as follows:

$$
\begin{gathered}
x_{i}=\left[X_{i}^{\alpha}+X_{i}^{\beta}+X_{i}^{\gamma}+X_{i}^{\delta}\right] / 4, \\
y_{m}=\left[X_{m}^{\eta}+X_{m}^{\kappa}+X_{m}^{v}+X_{m}^{\omega}\right] / 4,
\end{gathered}
$$

where $X_{i}^{\alpha}$ represents the probability that site $\alpha$ is occupied by either $B_{1}$ or $B_{2}$, and $X_{m}^{\eta}$ represents the probability that site $\eta$ is occupied by either $A_{1}$ or $A_{2}$.

The parameters $\mu_{B_{i}}^{*}$ and $\mu_{A_{m}}^{*}$ in Eq. (3) are the effective chemical potentials of $B_{i}$ species on the $I$ sublattice and of $A_{m}$ species on the $S$ sublattice, respectively. They are defined as

$$
\mu_{B_{i}}^{*}=\mu_{B_{i}}-\left(\mu_{B_{1}}+\mu_{B_{2}}\right) / 2, \quad \mu_{A_{m}}^{*}=\mu_{A_{m}}-\left(\mu_{A_{1}}+\mu_{A_{2}}\right) / 2,
$$

where $\mu_{B_{i}}$ and $\mu_{A_{m}}$ are the chemical potentials of component $B_{i}$ and $A_{m}$, respectively. The effective chemical potentials satisfy thus the relations $\mu_{B_{1}}^{*}+\mu_{B_{2}}^{*}=0$ and $\mu_{A_{1}}^{*}+\mu_{A_{2}}^{*}=0$. Note that in Eq. (3) the chemical potential is written separately for each sublattice, the factor $1 / 2$ ensures that each term contains only one half of the total number of lattice sites in the system. 


\section{A. Configurational entropy in the simple-cube approximation}

In the framework of the CVM, the configurational entropy is described as a function of cluster and subcluster distribution variables. The analysis of possible subclusters of the simple cube that contribute to the total configurational entropy (following the procedure described in Ref. 22) resulted in that only the points, the nearest-neighbor pairs, and the squares have nonzero contributions. Note that the nextnearest-neighbor pairs, although not appearing in the expression of the total entropy, contribute to the internal energy.

Following Kikuchi's procedure, ${ }^{5}$ the configurational entropy per lattice site in the simple-cube approximation becomes

$$
\begin{aligned}
S= & k_{B}[-L\{\text { cube }\}+3 L\{\text { square }\}-3 L\{\text { pair }\}+L\{\text { point }\}] \\
= & k_{B}\left[-\sum_{i j k l m n p s} L\left\{C_{i j k l m n p s}^{\alpha \beta \gamma \delta \eta \kappa v \omega}\right\}+\frac{1}{2}\left(\sum_{i j m n} L\left\{W_{i j m n}^{\alpha \beta \eta \kappa}\right\}\right.\right. \\
& +\sum_{i k n p} L\left\{W_{i k n p}^{\alpha \gamma \kappa v}\right\}+\sum_{i l m p} L\left\{W_{i l m p}^{\alpha \delta \eta \eta}\right\}+\sum_{j k n s} L\left\{W_{j k n s}^{\beta \gamma \kappa \omega}\right\} \\
& \left.+\sum_{j l m s} L\left\{W_{j l m s}^{\beta \delta \eta \omega}\right\}+\sum_{k l p s} L\left\{W_{k l p s}^{\gamma \delta v \omega}\right\}\right)-\frac{1}{4}\left(\sum_{i m} L\left\{Y_{i m}^{\alpha \eta}\right\}\right. \\
& +\sum_{i n} L\left\{Y_{i n}^{\alpha \kappa}\right\}+\sum_{i p} L\left\{Y_{i p}^{\alpha \eta}\right\}+\sum_{j m} L\left\{Y_{j m}^{\beta \eta}\right\}+\sum_{j n} L\left\{Y_{j n}^{\beta \kappa}\right\} \\
& +\sum_{j s} L\left\{Y_{j s}^{\beta \omega}\right\}+\sum_{k n} L\left\{Y_{k n}^{\gamma \kappa}\right\}+\sum_{k p} L\left\{Y_{k p}^{\gamma v}\right\}+\sum_{k s} L\left\{Y_{k s}^{\gamma \omega}\right\} \\
& \left.+\sum_{\operatorname{lm}} L\left\{Y_{l m}^{\delta \eta}\right\}+\sum_{l p} L\left\{Y_{l p}^{\delta v}\right\}+\sum_{l s} L\left\{Y_{l s}^{\delta \omega}\right\}\right) \\
& +\frac{1}{8}\left(\sum_{i} L\left\{X_{i}^{\alpha}\right\}+\sum_{j} L\left\{X_{j}^{\beta}\right\}+\sum_{k} L\left\{X_{k}^{\gamma}\right\}+\sum_{l} L\left\{X_{l}^{\delta\}}\right\}\right. \\
& \left.\left.+\sum_{m} L\left\{X_{m}^{\eta}\right\}+\sum_{n} L\left\{X_{n}^{\kappa}\right\}+\sum_{p} L\left\{X_{p}^{v}\right\}+\sum_{s} L\left\{X_{s}^{\omega}\right\}\right)\right],
\end{aligned}
$$

where $k_{B}$ is Boltzmann's constant and the function $L\{a\}$ $\equiv a \ln a$.

\section{B. Internal energy in the simple-cube approximation}

In CVM calculations using the simple-cube approximation, the internal energy of the system per lattice site is taken equal to the weighted sum of the energies for all the 256 possible configurations of the cube cluster, i.e.,

$$
U=\sum_{\text {sites }} \varepsilon_{i j k l m n p s}^{\alpha \beta \gamma \delta \eta \kappa v \omega} C_{i j k l m n p s}^{\alpha \beta \gamma \delta \eta \kappa v \omega}
$$

where $\varepsilon_{i j k l m n p s}^{\alpha \beta \gamma \delta \eta \kappa \omega}$ is the energy of a specific configuration on the simple cube that occurs with a probability given by the distribution variable $C_{i j k l m n p s}^{\alpha \beta \gamma \delta \eta \kappa v \omega}$. The energy of a specific configuration $\varepsilon_{i j k l m n p s}^{\alpha \beta \gamma \delta \eta \kappa v \omega}$ is approximated as the sum of pairwise interactions between the nearest- and the next-nearestneighbor lattice sites within the cube, and hence the expression of the internal energy becomes

$$
\begin{aligned}
U= & \frac{1}{4}\left(\sum_{i m} Y_{i m}^{\alpha \eta} \varepsilon_{i m}^{\alpha \eta}+\sum_{i n} Y_{i n}^{\alpha \kappa} \varepsilon_{i n}^{\alpha \kappa}+\sum_{i p} Y_{i p}^{\alpha v} \varepsilon_{i p}^{\alpha v}+\sum_{j m} Y_{j m}^{\beta \eta} \varepsilon_{j m}^{\beta \eta}\right. \\
& +\sum_{j n} Y_{j n}^{\beta \kappa} \varepsilon_{j n}^{\beta \kappa}+\sum_{j s} Y_{j s}^{\beta \omega} \varepsilon_{j s}^{\beta \omega}+\sum_{k n} Y_{k n}^{\gamma \kappa} \varepsilon_{k n}^{\gamma \kappa}+\sum_{k p} Y_{k p}^{\gamma v} \varepsilon_{k p}^{\gamma v} \\
& \left.+\sum_{k s} Y_{k s}^{\gamma \omega} \varepsilon_{k s}^{\gamma \omega}+\sum_{l m} Y_{l m}^{\delta \eta} \varepsilon_{l m}^{\delta \eta}+\sum_{l p} Y_{l p}^{\delta v} \varepsilon_{l p}^{\delta v}+\sum_{l s} Y_{l s}^{\delta \omega} \varepsilon_{l s}^{\delta \omega}\right) \\
& +\frac{1}{2}\left(\sum_{i j} Y_{i j}^{\alpha \beta} \varepsilon_{i j}^{\alpha \beta}+\sum_{i k} Y_{i k}^{\alpha \gamma} \varepsilon_{i k}^{\alpha \gamma}+\sum_{i l} Y_{i l}^{\alpha \delta} \varepsilon_{i l}^{\alpha \delta}+\sum_{j k} Y_{j k}^{\beta \gamma} \varepsilon_{j k}^{\beta \gamma}\right. \\
& \left.+\sum_{j l} Y_{j l}^{\beta \delta} \varepsilon_{j l}^{\beta \delta}+\sum_{k l} Y_{k l}^{\gamma \delta} \varepsilon_{k l}^{\gamma \delta}\right)+\frac{1}{2}\left(\sum_{m n} Y_{m n}^{\eta \kappa} \varepsilon_{m n}^{\eta \kappa}+\sum_{m p} Y_{m p}^{\eta v} \varepsilon_{m p}^{\eta v}\right. \\
& \left.+\sum_{m s} Y_{m s}^{\eta \omega} \varepsilon_{m s}^{\eta \omega}+\sum_{n p} Y_{n p}^{\kappa v} \varepsilon_{n p}^{\kappa v}+\sum_{n s} Y_{n s}^{\kappa \omega} \varepsilon_{n s}^{\kappa \omega}+\sum_{p s} Y_{p s}^{v \omega} \varepsilon_{p s}^{v \omega}\right),
\end{aligned}
$$

where the fractions $1 / 4$ and $1 / 2$ take care of the fact that the nearest-neighbor pairs and the next-nearest-neighbor pairs are shared between four and two cubes, respectively. Note that nearest-neighbor pairs occur between species occupying sites on different ( $S$ and $I$ ) sublattices, whereas next-nearestneighbor pairs are formed within the same $(S$ or $I)$ sublattice (Fig. 1). $\varepsilon_{i m}^{\alpha \eta}$ is the pairwise effective interaction between sublattice site $\alpha$ occupied with $i$ and sublattice site $\eta$ occupied with $m$. The so-called 8-4 type Lennard-Jones (L-J) potential $[\mathrm{Eq} .(8)]$ is used in this work to describe the volume dependence of the pair interactions, ${ }^{21}$

$$
\varepsilon_{i m}^{\alpha \eta}(r)=\varepsilon_{i m}^{0}\left[\left(\frac{r_{i m}^{0}}{r}\right)^{8}-2\left(\frac{r_{i m}^{0}}{r}\right)^{4}\right] .
$$

Equation (8) is written for the case of nearest-neighbor pairs consisting of an $I$ site and a $S$ site. Similar expressions including the proper indices can be written for the other types of pairs. In Eq. (5), $\varepsilon_{i m}^{0}$ and $r_{i m}^{0}$ are L-J parameters and $r$ is the distance between sites. In principle the L-J parameters can be derived based on thermodynamic data of cohesive energy, heats of formation, and lattice constants.

\section{Calculation of phase equilibrium}

For a given temperature $T$ and fixed effective chemical potentials $\mu_{B_{i}}^{*}$ (I sublattice) and $\mu_{A_{m}}^{*}(S$ sublattice $)$, the equilibrium state of the system corresponds to those configurations that minimize the value of the grand potential $\Omega\left(V, T, \mu_{B_{1}}^{*}, \mu_{B_{2}}^{*}, \mu_{A_{1}}^{*}, \mu_{A_{2}}^{*}\right)$. In this work, the natural iteration method $^{5,23}$ (NIM) is used to minimize the grand potential with respect to the cluster distribution variables. The minimization of $\Omega$ with respect to $C_{i j k l m n p s}^{\alpha \beta \gamma \delta \eta \kappa \omega}$ yields 


$$
\begin{aligned}
C_{i j k l m n p s}^{\alpha \beta \gamma \delta \eta v \omega}= & \exp \left(\frac{\lambda}{k T}\right) \exp \left(-\frac{\varepsilon_{i j k l m n p s}^{\alpha \beta \gamma \delta \eta \nu \omega}}{k T}\right) \\
& \times \exp \left(\frac{\mu_{B_{i}}^{*} X_{i}^{\alpha}+\mu_{B_{j}}^{*} X_{j}^{\beta}+\mu_{B_{k}}^{*} X_{k}^{\gamma}+\mu_{B_{l}}^{*} X_{l}^{\delta}}{8 k T}\right) \\
& \times \exp \left(\frac{\mu_{A_{m}}^{*} X_{m}^{\eta}+\mu_{A_{n}}^{*} X_{n}^{\kappa}+\mu_{A_{p}}^{*} X_{p}^{v}+\mu_{A_{s}}^{*} X_{s}^{\omega}}{8 k T}\right) \\
& \times\left(W_{i j k l m n p s}\right)^{1 / 2}\left(Y_{i j k l m n p s}\right)^{-1 / 4}\left(X_{i j k l m n p s}\right)^{1 / 8},
\end{aligned}
$$

with

$$
\begin{gathered}
W_{i j k l m n p s}=W_{i j m n}^{\alpha \beta \eta \kappa} W_{i k n p}^{\alpha \gamma \kappa v} W_{i l m p}^{\alpha \delta \eta v} W_{j k n s}^{\beta \gamma \kappa \omega} W_{j l m s}^{\beta \delta \eta \omega} W_{k l p s}^{\gamma \delta \omega \omega}, \\
Y_{i j k l m n p s}=Y_{i m}^{\alpha \eta} Y_{i n}^{\alpha \kappa} Y_{i p}^{\alpha v} Y_{j m}^{\beta \eta} Y_{j n}^{\beta \kappa} Y_{j s}^{\beta \omega} Y_{k n}^{\gamma \kappa} Y_{k p}^{\gamma v} Y_{k s}^{\gamma \omega} Y_{\operatorname{lm}}^{\delta \eta} Y_{l p}^{\delta v} Y_{l s}^{\delta \omega},
\end{gathered}
$$

and

$$
X_{i j k l m n p s}=X_{i}^{\alpha} X_{j}^{\beta} X_{l}^{\gamma} X_{l}^{\delta} X_{m}^{\eta} X_{n}^{\kappa} X_{p}^{v} X_{s}^{\omega},
$$

where $\lambda$ is the Lagrange multiplier taking into account the normalization conditions given in Eq. (1).

The volume per cluster site, $V$, corresponding to a particular configuration $C_{i j k l m n p s}^{\alpha \beta \gamma \delta \kappa v \omega}$ is obtained through Eq. (10) at constant $T$ and taking atmospheric pressure $p$ as reference,

$$
\left.\frac{\delta \Omega}{\delta V}\right|_{T, \mu^{*}, C_{i j k l m n p s}^{\alpha \beta \gamma \delta \eta \kappa \nu \omega}}=-p .
$$

The thermodynamic equilibrium is determined by the $T$, $\mu_{B_{i}}^{*}$, and $\mu_{A_{m}}^{*}$ for which $\Omega$ 's are the same for both phases. The concentrations and the lattice parameters of the phases in equilibrium are obtained from Eqs. (9) and (10).

\section{APPLICATION TO CALCULATIONS OF PHASE DIAGRAMS}

Phase diagram calculations were performed in order to demonstrate how the proposed simple-cube approximation of CVM accounts for the coupling between the substitutional and interstitial sublattices. The systems considered consist of a binary host alloy with interstitials, i.e., a fcc substitutional $A_{1}-A_{2}$ alloy which contains $B_{1}$ and $B_{2}$ interstitial species, were $B_{2}$ is taken as a vacancy. The cases investigated here are referred to as hypothetical, in view of the fact that the values of the input parameters are rather arbitrarily chosen and varied. The input parameters required for phase diagram calculations are the values of the Lennard-Jones pairinteraction parameters, i.e., $\varepsilon^{0}$ and $r^{0}$ [Eq. (8)]. Relative (normalized) values for the L-J parameters and $\mu^{*}$ with respect to a reference state are used. The L-J parameters of pure host $A_{1}$ are chosen as the reference state for all L-J parameters; and all the effective chemical potentials $\mu^{*}$ are normalized with respect to $\varepsilon_{A_{1} A_{1}}^{0}$. The values chosen for $\varepsilon_{A_{1} A_{1}}^{0}$ and $r_{A_{1} A_{1}}^{0}$ are $62.80 \mathrm{~kJ} / \mathrm{mol}(15 \mathrm{kcal} / \mathrm{mol})$ and $0.27 \mathrm{~nm}$, respectively. These values lie typically within the range of Lennard-Jones parameters for transition metals. ${ }^{17,21}$ One further remark has to be made regarding the energy $E$ and the entropy $S$. The vibrational contributions to energy and entropy are not considered in the calculations, since the main purpose of the
TABLE I. Normalized Lennard-Jones parameters $\varepsilon^{o}$ and $r^{o}$ used to model a $A_{1}-A_{2}-B_{1}-B_{2}$ system with the simple-cube approximation of CVM.

\begin{tabular}{lccccc}
\hline Pairs & \multirow{2}{*}{ Normalized parameters } & \multirow{2}{*}{ Pairs } & \multicolumn{2}{c}{ Normalized parameters } \\
\cline { 2 - 3 } \cline { 5 - 6 } & $\varepsilon^{0, \text { norm }}$ & $r^{0, \text { norm }}$ & & $\varepsilon^{0, \text { norm }}$ & $r^{0, \text { norm }}$ \\
\hline$A_{1}-A_{1}$ & 1.000 & 1.000 & $B_{2}-B_{2}$ & 0.000 & 1.000 \\
$A_{1}-A_{2}$ & 0.980 & 1.050 & $A_{1}-B_{1}$ & 0.010 & 1.280 \\
$A_{2}-A_{2}$ & 0.950 & 1.080 & $A_{2}-B_{1}$ & 0.008 & 1.330 \\
$B_{1}-B_{1}$ & 0.021 & 1.280 & $A_{1}-B_{2}$ & 0.000 & 1.000 \\
$B_{1}-B_{2}$ & 0.036 & 1.230 & $A_{2}-B_{2}$ & 0.000 & 1.000 \\
\hline \hline
\end{tabular}

present work is to qualitatively demonstrate how the proposed formalism describes the coupling of $S$ and $I$ sublattices and to show the effect of that on phase diagrams.

The CVM calculations are performed, at constant (atmospheric) pressure, in the three-dimensional parameter space $\left(T, \mu_{B}^{*}, \mu_{A}^{*}\right)$. The results discussed here are obtained from calculations using the normalized parameters given in Table I. The interstitial species $B_{2}$ are taken as vacancies that only interact with the interstitial species $B_{1}$.

First, the value of the effective chemical potentials $\mu_{A_{1}}^{*}\left(=-\mu_{A_{2}}^{*}\right)$ on the $S$ (host) sublattice is fixed. Note that fixing the value for $\mu_{A_{1}}^{*}$ implies that the tangent to the Gibbs energy of the host lattice is fixed (see Appendix A of Ref. 17) at the same value for all $T$ used in the calculations. Since the shape of the Gibbs energy varies with $T$, this implies that the composition of the host matrix for which the calculation is performed differs for each $T$. The equilibrium state is searched for a given temperature $T$ by varying the effective chemical potentials on the $I$ sublattice $\mu_{B_{1}}^{*}\left(=-\mu_{B_{2}}^{*}\right)$; see Fig. 2 . The phase equilibrium is determined by the $T$ and $\mu_{B_{1}}^{*}$ for which $\Omega$ is the same for both phases, i.e., it corresponds to the intersection of the two curves each representing the variation of the grand potential of a phase with $\mu_{B_{1}}^{*}$,norm $\left(=\mu_{B_{1}}^{*} / \varepsilon_{A_{1} A_{1}}^{0}\right){ }^{23}$ The composition of the phases at equilibrium (at $T=400 \mathrm{~K}, \mu_{A_{1}}^{* \text {,norm }}=0$, and $\mu_{B_{1}}^{*, \text { norm }}=1.464$ ) is obtained from the corresponding site occupancies. The calculations at given fixed $\mu_{A_{1}}^{*, \text { norm }}$ are repeated for different $T$ in order to obtain the equilibrium temperature-composition diagrams shown in Fig. 3. Note that the compositions in Fig. 3 are given in terms of the mole fractions for each sublattice, as defined in Eq. (4), and not in terms of overall mole fractions. The equilibrium compositions of the $A_{1}-A_{2}$ host alloy as function of $T$ are given in the left graphs of Fig. 3. The corresponding compositions of the interstitial sublattice for these phases in equilibrium are given in the graphs at the right.

The temperature-composition diagrams for different values of $\mu_{A_{1}}^{* \text {,norm }}$ of the host, namely $1.0,0.0,-1.0$, are given in Fig. 3. The diagrams in Fig. 3 show the phase boundaries on the $S$ sublattice (left) and the corresponding compositions of the $I$ sublattice (right) as calculated in the temperature range $(200-1400 \mathrm{~K})$.

The diagrams in Fig. 3 indicate first of all that significantly different phases exist for different values of $\mu_{A_{1}}^{*, \text { norm }}$. 


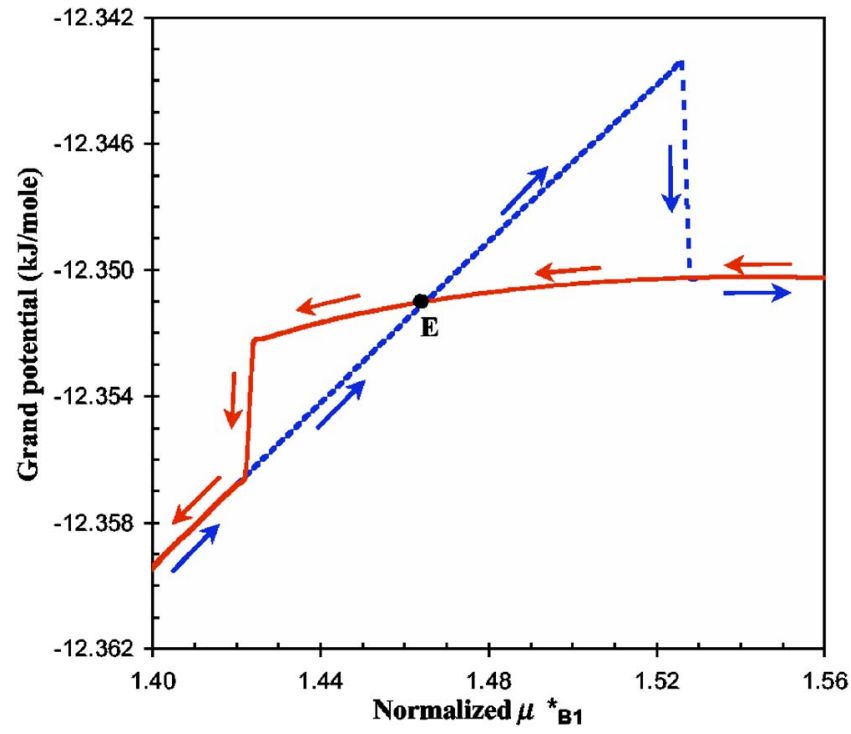

FIG. 2. (Color online) The grand potential as function of the effective chemical potential on the $I$ sublattice $\left(\mu_{B_{1}}^{*, n o r m}\right)$ at fixed temperature $(T=400 \mathrm{~K})$ and fixed effective chemical potential on the $S$ sublattice $\left(\mu_{A_{1}}^{*, n o r m}=0\right)$. The equilibrium state is searched for by firstly increasing $\mu_{B_{1}}^{*}$,norm (dotted curve) and then by decreasing $\mu_{B_{1}}^{*, n o r m}$ (solid curve) as indicated by arrows along the curves. The equilibrium state corresponds to point $E\left(\mu_{B_{1}}^{*, n o r m}=1.464\right)$.

For instance, for $\mu_{A_{1}}^{*, \text { norm }}=1.0$ the host sublattice $S$ is occupied exclusively by $A_{1}$ over the whole temperature range whereas this is not the case for $\mu_{A_{1}^{*}}^{*, n o r m}=0$ and -1 (see diagrams at the left in Fig. 3 ). For the case $\mu_{A_{1}^{*}}^{* \text { norm }}=1$, a coexistence region with a critical temperature around $510 \mathrm{~K}$ exists between two phases which dissolve different amounts of interstitials.

In all cases the composition of the $I$ sublattice depends strongly on the compositions of the substitutional host sublattice. For the case of $\mu_{A_{1}}^{*}$,norm $=-1.0$, at any temperature between $200 \mathrm{~K}$ and $1200 \mathrm{~K}$, at equilibrium, the system consists of a $A_{1}$-rich phase and a $A_{1}$-poor phase which dissolve different amounts of $B_{1}$ interstitials. The $A_{1}$-poor phase contains a larger amount of interstitials $B_{1}$ than the $A_{1}$-rich phase (the compositions of both sublattices of one phase are denoted with the same marks in the left and right graphs). For the case $\mu_{A_{1}}^{*, \text { norm }}=0$ the temperature composition diagram is a combination of the two other cases. At low temperatures the diagram for $\mu_{A_{1}}^{*, \text { norm }}=0$ is similar to the case of $\mu_{A_{1}}^{*, \text { norm }}=1.0$. Then, around $T=510 \mathrm{~K}$, the two solid phases in equilibrium have a quite different content of $A_{1}$ and $A_{2}$. Again, the $A_{1}$-rich phase, which contains less than $10 \% A_{2}$ on the $S$ sublattice, dissolves a lower amount of $B_{1}$ interstitials, but the solubility is larger than in the case $\mu_{A_{1}^{*}}^{*}$,norm $=1.0$ and lower than in the case $\mu_{A_{1}}^{*, \text { norm }}=-1.0$.

The results presented in Fig. 3 differ only in the value of the chemical potential of species on the host sublattice (different $\mu_{A_{1}}^{*, \text { norm }}$ ) as the starting point for the calculations. The obtained equilibrium compositions on the $I$ sublattice are strongly coupled to those of the $S$ sublattice.

Next, a set of CVM calculations were performed for fixed temperature $T$ and fixed chemical potentials of the interstitial species $\mu_{B_{1}}^{*}\left(=-\mu_{B_{2}}^{*}\right)$; this implies that the tangent to the Gibbs energy of the interstitial lattice is fixed. As for the previous case, the composition (here) of the interstitial lattice is fixed at each $T$; since the shape of the Gibbs energy could change with $T$ also the composition could change. The equilibrium phase boundaries are then determined by varying $\mu_{A_{1}}^{*}$. The phase diagrams in the $\left(T, y_{1}\right)$ and $\left(T, x_{1}\right)$ planes are shown in Fig. 4 for $\mu_{B_{1}}^{*, \text { norm }}=1.0,0.0$, and -1.0 , respectively. In all these cases, the system separates into two phases, one $A_{1}$-rich phase (represented by solid lines in Fig. 4) and one $A_{1}$-poor (represented by dotted lines in Fig. 4), which dissolve a lower or larger amount of $B_{1}$ interstitials, respectively. This suggests that the presence of $B_{1}$ interstitial in a $A_{1}-A_{2}$ host system induces phase separation. With decreasing the value of $\mu_{B_{1}}^{*, n o r m}$ the critical temperature of the twophase region increases. These temperature-composition diagrams demonstrate again that phase transitions on the $S$ and $I$ sublattices of these fcc alloys are strongly coupled to each other.

From a practical point of view, the chemical potential is a parameter rather difficult to control or estimate directly from experiments. In this sense, Figs. 3 and 4 are less suitable because they apply to cases of fixed chemical potential, and variable composition of both the metal host alloy and the interstitial sublattices. More useful are phase diagram representations that give direct information about the composition of the phases coexisting in a system at given $T, p$, and overall composition. Hence, representations in the composition plane $\left(x_{i}, y_{m}\right)$ at constant temperature $T$ and $p$, which are similar with the so-called Gibbs triangle for ternary systems, are preferable. Examples of such diagrams for the system studied in this paper are given in Figs. 5(a) and 5(b) at temperatures of $400 \mathrm{~K}$ and $800 \mathrm{~K}$, respectively. In these cases, the CVM calculations were performed at constant $T$ (and $p$ ) by varying the $\mu_{A_{1}^{*} \text {,norm }}$ and searching the corresponding equilibrium $\mu_{B_{1}}^{*, \text { norm }}$ value (and vice versa), so to obtain a set of equilibrium points in the $\left(\mu_{A_{1}}^{*, \text { norm }}, \mu_{B_{1}}^{*, \text { norm }}\right)$ space. In Fig. 5 the compositions of the phases in equilibrium are represented by tie lines (i.e., lines connecting two points of identical effective chemical potentials). A system with an overall composition represented by a point in the miscibility gap [e.g., a system with overall composition of $y_{1}=0.80\left(A_{1}\right)$ and $x_{1}$ $=0.70\left(B_{1}\right)$, represented by point $C$ in Fig. 5(a)] separates into two phases that coexist. The compositions of these two phases correspond to the ends of the tie line drawn through the point [e.g., at $400 \mathrm{~K}$ the composition of phase $\mathrm{I}$ is $y_{1}$ $=0.60\left(A_{1}\right)$ and $x_{1}=0.78\left(B_{1}\right)$ and that of phase II is $y_{1}$ $=0.99$ and $\left.x_{1}=0.61\right]$. With increasing temperature, the miscibility gap decreases.

CVM allows one to evaluate the presence of short- and long-range order. ${ }^{10-17}$ In the present calculations it was assumed that both the $S$ and $I$ sublattices of a fcc system exhibit a disordered $(A 1)$ structure, i.e., the starting symmetry of the structure was described by taking $X_{m}^{\eta}=X_{n}^{\kappa}=X_{p}^{v}=X_{s}^{\omega}$ for $m=n=p=s$ equal to 1 or 2 (on the $S$ sublattice) and $X_{i}^{\alpha}$ $=X_{j}^{\beta}=X_{k}^{\gamma}=X_{l}^{\delta}$ for $i=j=k=l$ equal to 1 or 2 (on the $I$ sublattice). Hence, from the present calculations it is possible to evaluate whether short-range ordering (SRO) occurs in the 

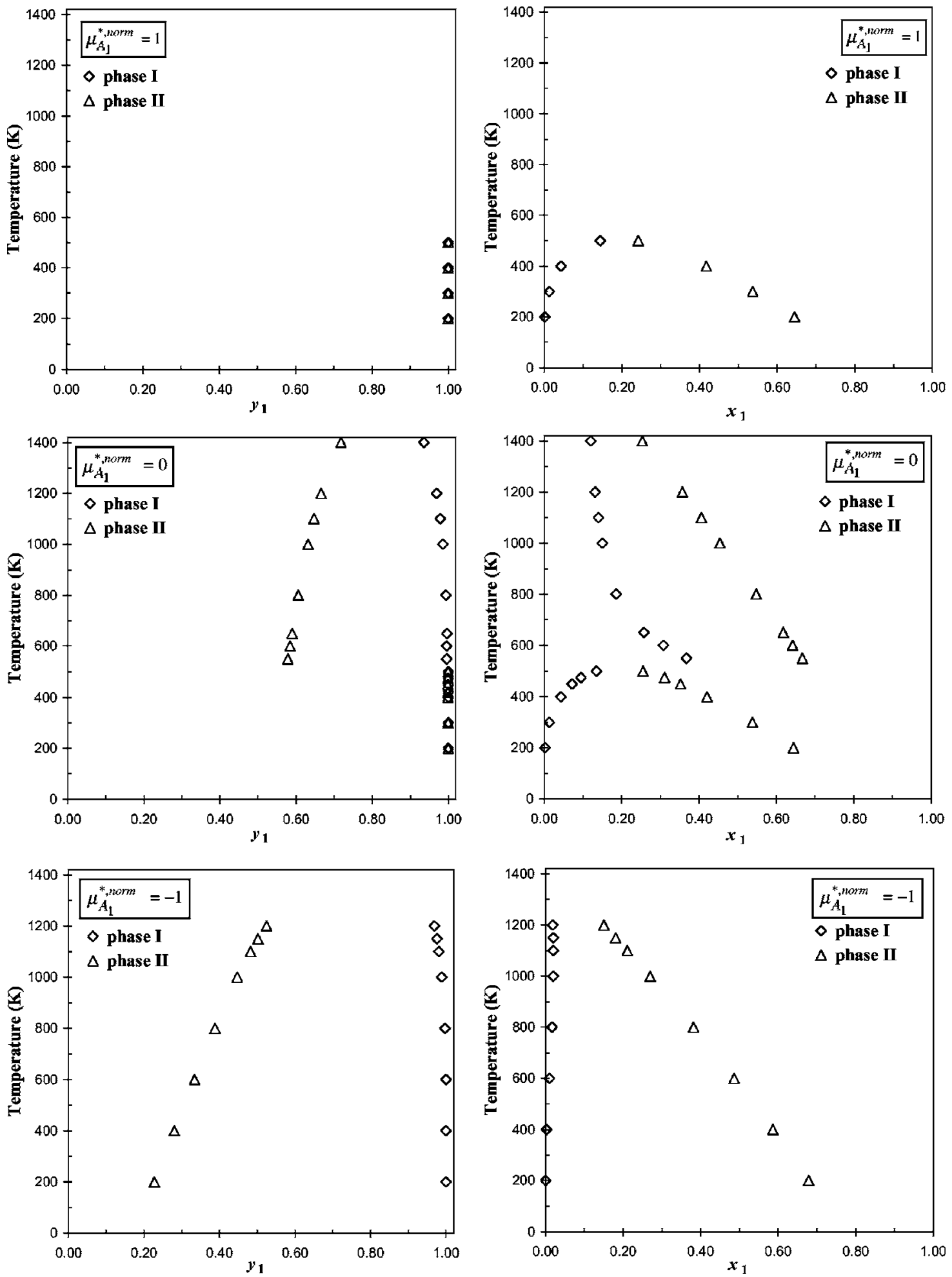

FIG. 3. Coupled phase diagrams of a hypothetical fcc system, as calculated from the simple-cube approximation using parameters given in Table I. The effective chemical potential on the substitutional sublattice $\mu_{A_{1}}^{*, n o r m}$ was fixed during the calculations. Figures on the same row are obtained from the same calculations; the left and right figures are for the substitutional and interstitial sublattices, respectively.

system. This can be achieved by comparing the site occupancies of the cube clusters calculated for a fixed composition and temperature from the cluster distribution variables $C_{i j k l m n p s}^{\alpha \beta \gamma \delta \eta \omega}$ obtained by CVM, with those corresponding to a random distribution of atoms at the same composition. How- ever, since the equilibrium phase boundaries for the systems discussed here are given as temperature-composition diagrams for both (the substitutional and the interstitial) sublattices (Figs. 3 and 4), it is convenient to analyze the site occupancies on each sublattice (i.e., the occupation of the 

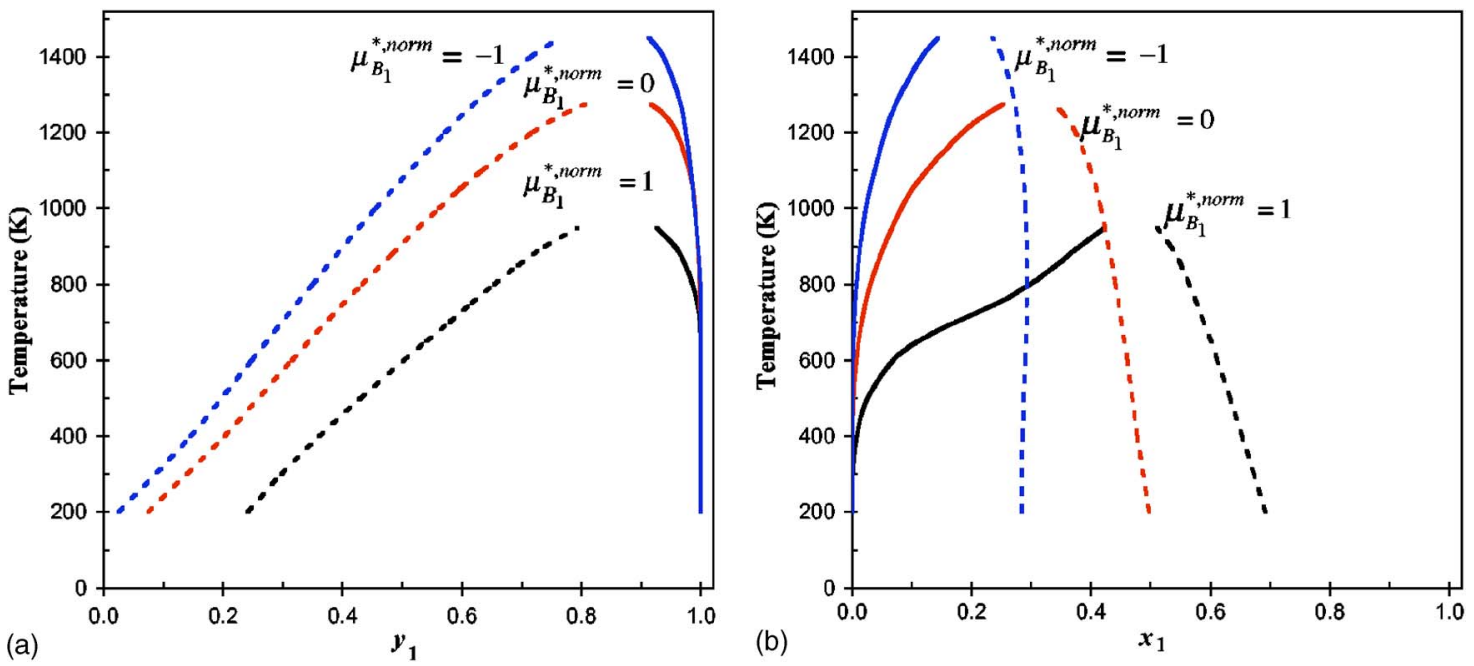

FIG. 4. (Color online) Coupled phase diagrams of a hypothetical fcc system, as calculated from the simple-cube approximation using parameters given in Table I. During the calculations the effective chemical potential on the interstitial sublattice $\mu_{B_{1}}^{*, \text { norm }}$ was fixed. (a) and (b) are obtained from the same calculations and correspond to the substitutional and interstitial sublattices, respectively. Dotted lines, phase I; solid lines, phase II.

two tetrahedrons comprising the cube cluster; see Fig. 1). These site occupancies are obtained from the cube-cluster distribution variables by taking the appropriate summations [Eq. (2)].

The analysis of the site occupations of the two tetrahedron clusters comprising the cube cluster has shown that the solid phases in equilibrium at given conditions of $T, p$, and chemical potentials, exhibit different degrees of SRO. For example, for the cases presented in Fig. 3, at $400 \mathrm{~K}$, the two phases in equilibrium (phase I and phase II) show random distribution of atoms on both, the $S$ and the $I$ sublattices for $\mu_{A_{1}}^{*, \text { norm }}=0$ or 1.0. However, this is not the case for $\mu_{A_{1}}^{*}$,norm $=-1.0$, where some SRO occurs in phase II. The fractions of

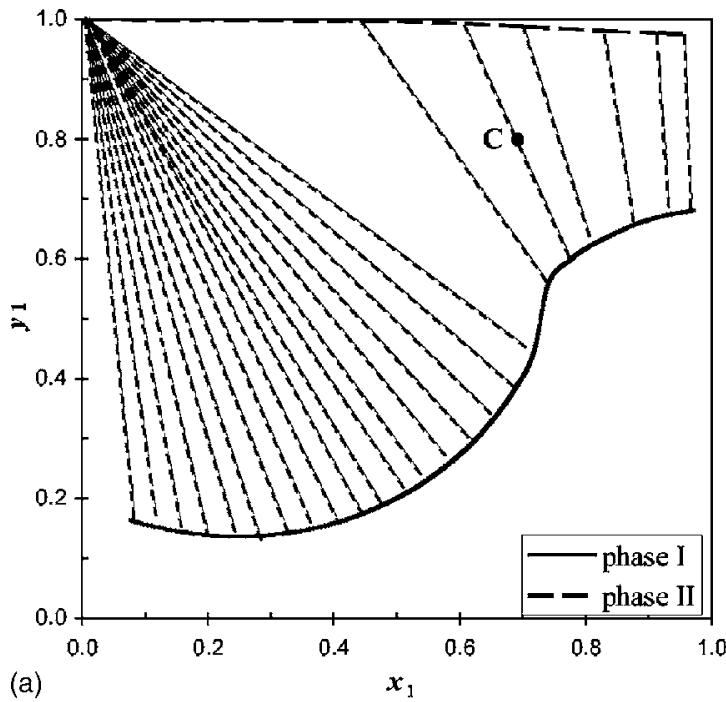

$S$ and $I$ tetrahedron clusters within the cubes, occupied by 0 to 4 atoms $A_{1}$ ( $S$ sublattice) or $B_{1}$ ( $I$ sublattices), calculated by CVM with $\mu_{A_{1}^{*}}^{* \text { norm }}=-1$ for phase II in equilibrium with phase I at $400 \mathrm{~K}$ are shown in Fig. 6. The occupations corresponding to random distributions at the corresponding compositions are also shown for comparison. A clear difference between CVM results and the random distribution is observed, i.e., according to the CVM calculations a larger fraction of $S$ tetrahedron clusters is occupied with only one or two $A_{1}$ atoms, a smaller fraction is empty, and only a small fraction contains three or four $A_{1}$ atoms; on the $I$ sublattice of the same phase, a larger fraction of $I$ tetrahedrons is occupied with two or three $B_{1}$ atoms, and a smaller fraction

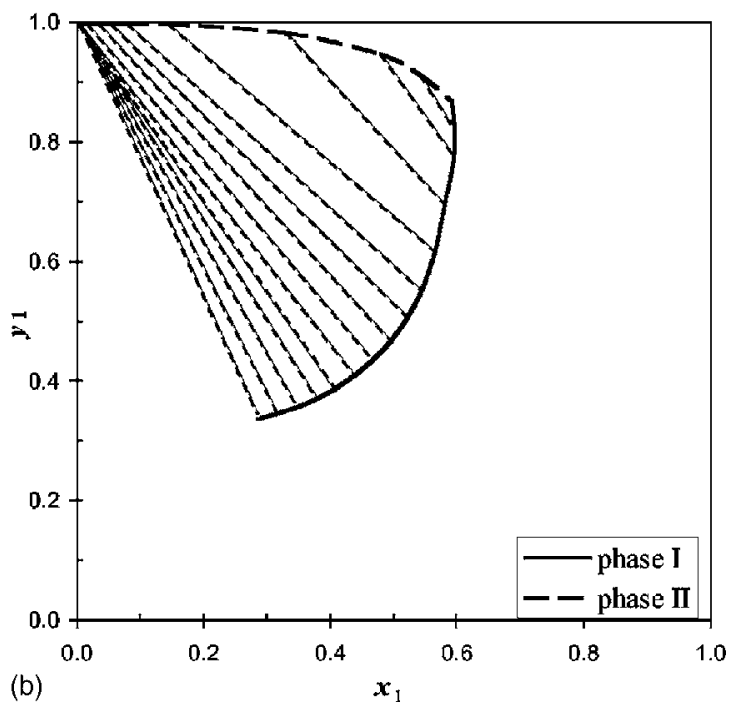

FIG. 5. Miscibility gap in the $\left(x_{1}, y_{1}\right)$ plane for the $A_{1}-A_{2}$ host alloy with $B_{1}$ interstitial species. The temperature is (a) $T=400 \mathrm{~K}$ and (b) $800 \mathrm{~K}$. The dotted lines connect two points where the chemical potentials are identical (tie lines). For example, a system with an overall composition represented by point $C$ in (a) with overall composition $y_{1}=0.80\left(A_{1}\right), x_{1}=0.70\left(B_{1}\right)$ separates into two phases that coexist. The compositions of these two phases correspond to the ends of the tie line drawn through the point [i.e., for phase I $y_{1}=0.60\left(A_{1}\right), x_{1}=0.78\left(B_{1}\right)$ and for phase II, $\left.y_{1}=0.99, x_{1}=0.61\right]$. 
is empty or occupied by one or four $B_{1}$ atoms. The site occupancies of the two sublattices are coupled, i.e., higher fractions of $I$ tetrahedrons occupied by two or more $B_{1}$ atoms correspond to lower fractions of $S$ tetrahedrons within the cube clusters occupied by two or more $A_{1}$ atoms. This is in agreement with the previous remark that the composition of the $I$ sublattice depends strongly on the compositions of the substitutional host sublattice (and vice versa), and that the $A_{1}$-poor phases dissolve larger amounts of $B_{1}$ interstitials.

\section{CONCLUSIONS}

The above CVM calculations using the simple-cube approximation demonstrate that phase transitions and ordering transitions on the $S$ and $I$ sublattices of these hypothetical fcc alloys are strongly coupled to each other, and that this mutual interaction can be described by the proposed simple-cube approximation of the CVM. This phenomenon has already been observed experimentally in some systems, ${ }^{1-4}$ but not accounted for in CVM modeling.

To apply this method to describing real systems, two routes can be followed to estimate the internal energy, i.e., (1) via pair interactions or (2) through first-principle calculations. For the first approach, the pair interaction parameters used in expressing the internal energy of the system should be obtained. To derive all these parameters (see Table I) based on experimental data (i.e., cohesive energies, lattice parameters) is quite tedious, if not impossible. Therefore, the use of internal energy expressed in terms obtained through $a b$ initio calculations could be a better approach. In that case, effective cluster interactions (ECI), which can be obtained from first-principle calculations, can be used. In previous work, ${ }^{20}$ some of us showed that the CVM-ECI approach is able to provide a reliable description of the phase boundaries in iron-nitrogen and iron-carbon-nitrogen systems. We believe the use of ECI's in the simple-cube approximation of

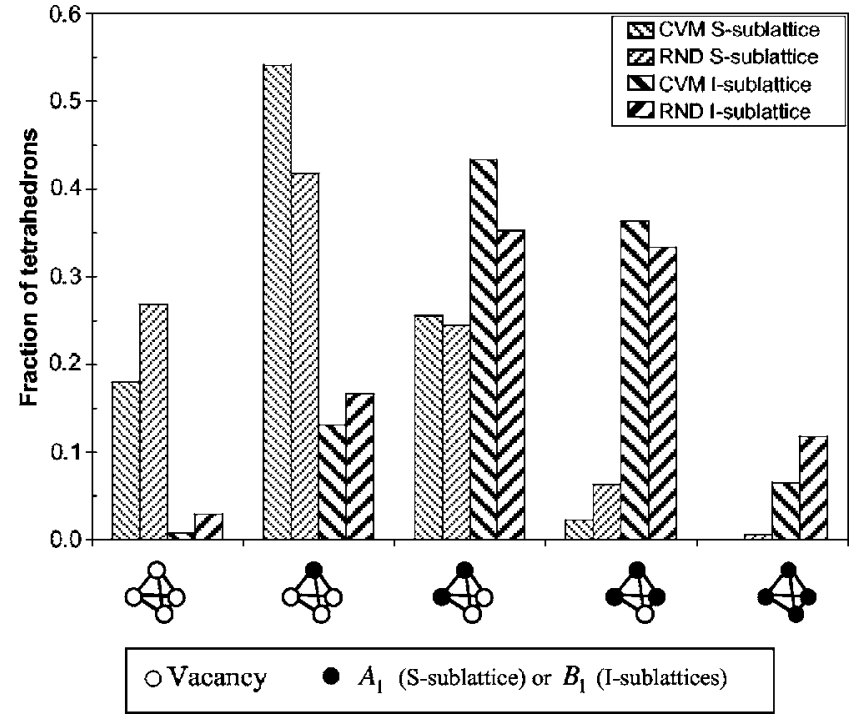

FIG. 6. The occupation of phase II at $400 \mathrm{~K}$; the fraction of tetrahedron clusters within cubes occupied by zero to four atoms $A_{1}$ ( $S$ sublattice) or $B_{1}$ ( $I$ sublattices), as calculated by CVM with $\mu_{A_{1}}^{*, \text { norm }}=-1$, and the random distributions at the corresponding compositions.

CVM could provide a good description of order-disorder phase transitions in fcc substitutional systems in the presence of interstitial atoms.

\section{ACKNOWLEDGMENTS}

This work is part of a research program of the Netherlands Institute for Metals Research (NIMR) and the Stichting voor Fundamenteel Onderzoek der Materie [FOM, financially supported by the Nederlandse Organisatie voor Wetenschappelijk Onderzoek (NWO)].
*On leave from the Delft University of Technology.

Corresponding author. Electronic address: A.J.Bottger@tudelft.nl

${ }^{1}$ H. Noh, T. B. Flanagan, B. Cerundolo, and A. Craft, Scr. Metall. Mater. 25, 225 (1991).

${ }^{2}$ T. B. Flanagan and Y. Sakamoto, Platinum Met. Rev. 37, 26 (1993).

${ }^{3}$ S. M. Lee, T. B. Flanagan, and G. H. Kim, Scr. Metall. Mater. 32, 827 (1994).

${ }^{4}$ P. Villars, A. Prince, and H. Okamoto, Handbook of Ternary Alloy Phase Diagrams, Vols. 3, 6, 8 (ASM International, Metals Park, $\mathrm{OH}, 1995)$.

${ }^{5}$ R. Kikuchi, Phys. Rev. 81, 988 (1951).

${ }^{6}$ D. de Fontaine, in Solid State Physics, edited by H. Ehrenreich and D. Turnbull (Academic Press, New York, 1994), Vol. 47, pp. 33-176.

${ }^{7}$ R. Kikuchi, J. Chem. Phys. 60, 1071 (1974).

${ }^{8}$ R. Kikuchi, J. M. Sanchez, D. de Fontaine, and H. Yamauchi, Acta Metall. 28, 651 (1980).
${ }^{9}$ J. M. Sanchez and D. de Fontaine, Phys. Rev. B 25, 1759 (1982).

${ }^{10}$ B. Burton and R. Kikuchi, Am. Mineral. 69, 165 (1984).

${ }^{11}$ J. M. Sanchez, J. R. Barefoot, R. N. Jarrett, and J. K. Tien, Acta Metall. 32, 1519 (1984).

${ }^{12}$ T. Mohri, J. M. Sanchez, and D. de Fontaine, Acta Metall. 33, 1171 (1985).

${ }^{13}$ M. Enomoto and H. Harada, Metall. Trans. A 20A, 649 (1989).

${ }^{14}$ C. Colinet, G. Inden, and R. Kikuchi, Acta Metall. Mater. 41, 1109 (1993).

${ }^{15}$ D. de Fontaine and C. Wolverton, Ber. Bunsenges. Phys. Chem. 96, 1503 (1992).

${ }^{16} \mathrm{C}$. Colinet, in Theory and Applications of the Cluster Variation Method and Path Probability Methods, edited by J. L. MoránLópez and J. M. Sanchez (Plenum Press, New York, 1996), p. 313.

${ }^{17}$ M. I. Pekelharing, A. J. Böttger, M. A. J. Somers, and E. J. Mittemeijer, Metall. Mater. Trans. A 30A, 1945 (1999).

${ }^{18}$ M. I. Pekelharing, A. J. Böttger, and E. J. Mittemeijer, Philos. Mag. 83, 1775 (2003). 
${ }^{19}$ S. Shang and A. J. Böttger, Acta Mater. 51, 3597 (2003).

${ }^{20}$ S. Shang and A. J. Böttger, Acta Mater. 55, 255 (2005).

${ }^{21}$ J. M. Sanchez, J. R. Barefoot, R. N. Jarrett, and J. K. Tien, Acta Metall. 32, 1519 (1984).
${ }^{22}$ J. M. Sanchez and D. de Fontaine, Phys. Rev. B 17, 2926 (1978).

${ }^{23}$ R. Kikuchi and D. de Fontaine, Proceedings of the NBS Workshop Application of Phase Diagrams in Metallurgy and Ceramics (Gaithersburg, 1977), p. 967. 